\title{
Esclerosis tuberosa: hallazgos clínicos e imagenológicos en una serie de pacientes pediátricos colombianos
}

\section{Tuberous sclerosis: clinical and imaging findings in a series of Colombian pediatric patients}

\author{
Divahía García-Martín,* Natalia Martínez-Córdoba, ${ }^{\ddagger}$ Paola Andrea-Cubides, * \\ Eugenia Teresa Espinosa-García, ${ }^{\S}$ Andrés Felipe Araujo-Polainaף \\ * Residente de Neurología Pediátrica. Universidad Militar Nueva Granada; ${ }^{\ddagger}$ Universidad Militar Nueva Granada; \\ § Servicio de Neurología Pediátrica, Hospital Militar Central. Universidad Militar Nueva Granada y Universidad \\ del Rosario; ` Servicio de Neurología Pediátrica, Hospital Militar Central. Bogotá, Colombia.
}

\begin{abstract}
RESUMEN
Introducción: El complejo esclerosis tuberosa (CET) es un trastorno autosómico dominante con afección multisistémica. Clínicamente se encuentran anormalidades en piel, ojo, sistema nervioso central (SNC), riñón, corazón y pulmón. Objetivo: Describir las características clínicas de pacientes pediátricos con CET. Material y métodos: Revisión retrospectiva de 12 pacientes con diagnóstico de CET atendidos en 10 años (20102020) en un hospital de tercer nivel de Bogotá, Colombia, en el que se analizan variables clínicas y de imagen de resonancia magnética (IRM). Resultados: Siete pacientes fueron mujeres. La edad media al diagnóstico fue de 16 meses. Sólo en tres tenían antecedente de un familiar de primer grado con la misma entidad. Los 12 pacientes presentaron alteración dermatológica, 10/12 (83\%) de los pacientes mostraron algún tipo de alteración cognitivo-conductual, $7 / 12$ presentaban crisis epilépticas y 5/12 alteraciones oftalmológicas. En 11/12 pacientes en IRM se evidenció alguna anormalidad, como nódulos subependimarios y displasia cortical. Conclusiones: El diagnóstico de CET está basado en criterios clínicos, incluyendo principalmente problemas neurológicos y dermatológicos. En estos pacientes se requiere de manejo interdisciplinario, dado que es una enfermedad multisistémica.
\end{abstract}

Palabras clave: Complejo esclerosis tuberosa, epilepsia, facomatosis, hamartomas, niños, adolescentes.

\begin{abstract}
Introduction: Tuberous sclerosis complex (TSC) is an autosomal dominant disorder with multisystem involvement. In this disease, abnormalities are found in the skin, eye, central nervous system (CNS), kidney, heart, and lung. Objective: To describe the clinical characteristics of pediatric patients with TSC. Material and methods: Retrospective review of 12 patients diagnosed with TSC treated over a 10-year period (2010-2020) in a tertiary hospital in Bogotá, Colombia. Clinical and magnetic resonance imaging (MRI) data were recorded. Results: Seven patients were women. The mean age at diagnosis was 16 months. Only three patients had a history of a first-degree relative with the same condition. The 12 patients had dermatological alterations, 10/12 (83\%) patients presented some type of cognitive-behavioral disorder, 7/12 with seizures, and 5/12 ophthalmological problems. In $11 / 12$ patients, the MRI showed some abnormality, such as subependymal nodules and cortical dysplasia. Conclusions: The diagnosis of TSC is based on clinical criteria, mainly including neurological and dermatological disorders. In these patients, interdisciplinary management is required, since it is a multisystemic disease.
\end{abstract}

Keywords: Tuberous sclerosis complex, epilepsy, phakomatosis, hamartomas, children, adolescent.

Correspondencia: Divahía García-Martín, divahia.garcia@gmail.com

Citar como: García-Martín D, Martínez-Córdoba N, Andrea-Cubides P, Espinosa-García ET, Araujo-Polaina AF. Esclerosis tuberosa: hallazgos clínicos e imagenológicos en una serie de pacientes pediátricos colombianos. Rev Mex Pediatr. 2021; 88(1): 10-17. https://dx.doi. org/10.35366/99413 


\section{INTRODUCCIÓN}

Los síndromes neurocutáneos o facomatosis son un grupo heterogéneo de enfermedades de carácter hereditario con afectación multisistémica que incluye lesiones a nivel cutáneo y del SNC. ${ }^{1}$ Entre el grupo de las facomatosis más comunes se encuentran la esclerosis tuberosa, la neurofibromatosis tipo 1 y 2 , el síndrome de Von Hippel-Lindau y la enfermedad de Sturge-Weber. ${ }^{2}$

El complejo esclerosis tuberosa (CET) es una enfermedad genética, autosómica dominante con expresividad variable; la prevalencia a nivel mundial es de 6.812.4 por 100,000 personas, su incidencia al nacimiento es de uno en 5,000 a 10,000 recién nacidos vivos, no encontrándose diferencias entre sexos o etnias. ${ }^{3}$ Fue descrita hace más de 150 años por Von Recklinghausen, quien describió por primera vez los esclerodemas del cerebro; sin embargo, Bourneville en 1880 publica la primera descripción de la enfermedad relacionándola con lesiones cerebrales y en la piel de la cara. ${ }^{1}$

Recientemente se han localizado dos genes causantes: el gen TSC1 (ubicado en el cromosoma 9q34, encargado de codificar la hamartina) y el gen TSC2 (ubicado en el cromosoma 16p 13.3, que codifica la tuberina). Estas proteínas actúan como supresores en el crecimiento de tumores, regulan la diferenciación y proliferación celular. ${ }^{4}$

El diagnóstico se basa en una serie de criterios clínicos y radiológicos, los cuales se agrupan en los criterios mayores y menores de acuerdo con lo señalado en la Tabla 1.5
A nivel cerebral se observan malformaciones causadas por alteraciones en la histogénesis, proliferación y migración de las neuronas y células gliales, donde se incluyen los nódulos subependimarios, túberes corticales, subcorticales y astrocitomas subependimarios de células gigantes (SEGA). ${ }^{6}$ Estas lesiones estructurales suelen estar asociadas a alteraciones neurológicas, como epilepsia, trastornos del espectro autista, de la conducta y la discapacidad intelectual. ${ }^{7}$

Debido a lo limitado de la información en América Latina, el objetivo del presente trabajo es describir las características clínicas e imagenológicas de 12 pacientes pediátricos con diagnóstico de complejo esclerosis tuberosa.

\section{MATERIAL Y MÉTODOS}

Se revisaron los casos de los pacientes con diagnóstico de CET entre 2010 y 2020, valorados por el Servicio de Neurología Pediátrica del Hospital Militar Central ubicado en la ciudad de Bogotá, Colombia. Este hospital corresponde a un tercer nivel de atención, el cual es un centro de referencia en el régimen especial (Fuerzas Militares), con cobertura total en el sistema de salud.

Se incluyeron pacientes con diagnóstico definitivo de CET, de acuerdo con los criterios del consenso internacional de 2012, con presencia de dos criterios mayores, o bien uno mayor y dos menores. ${ }^{5}$

Se recogieron las siguientes variables clínicas: edad, sexo, edad de diagnóstico del CET, edad actual, edad de inicio de epilepsia, tipo de crisis, lesiones dermatológi-

Tabla 1: Criterios diagnósticos del complejo esclerosis tuberosa.

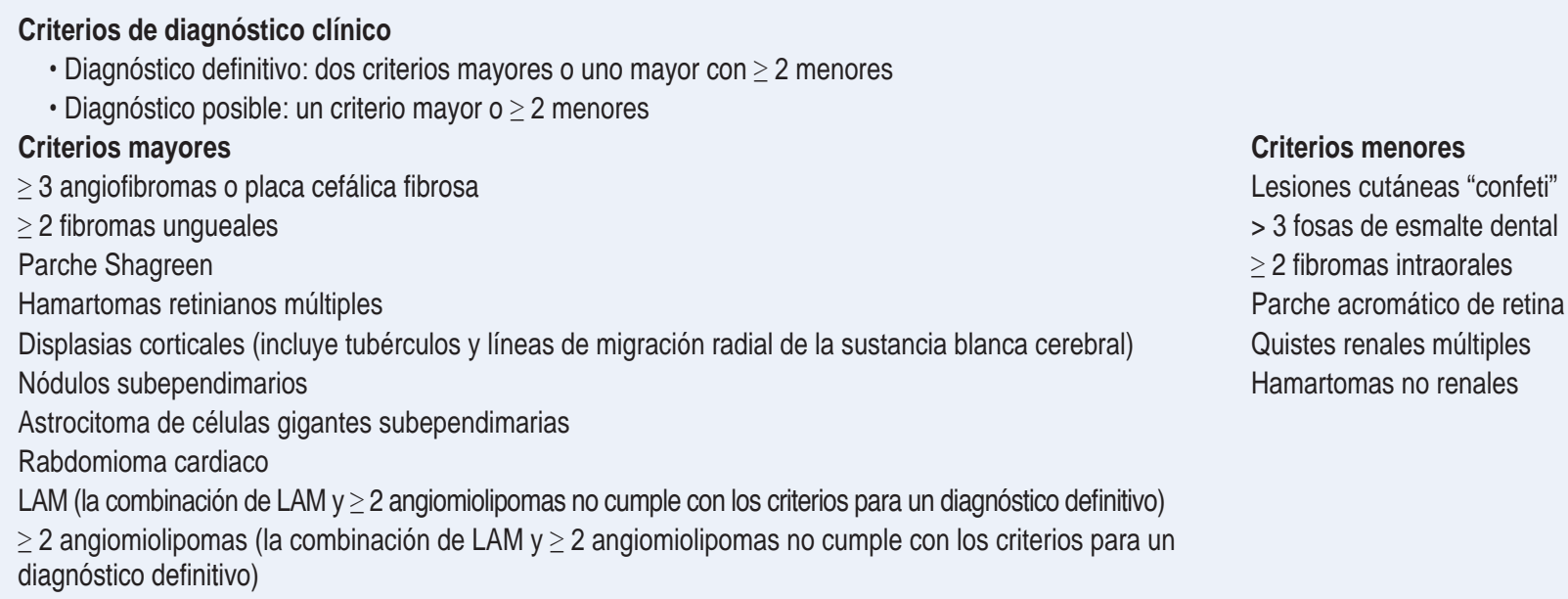

\section{Criterios menores}

Lesiones cutáneas "confeti" $>3$ fosas de esmalte dental $\geq 2$ fibromas intraorales Parche acromático de retina Quistes renales múltiples Hamartomas no renales 
cas, fármacos antiepilépticos que recibían, alteraciones neurológicas asociadas, trastornos del comportamiento, antecedentes familiares y prenatales. Además, se analizaron los hallazgos en el examen físico, de la exploración neurológica, de la resonancia magnética cerebral, ecografía renal, ecocardiograma y fondo de ojo.

En general, la valoración de los pacientes descritos ha sido interdisciplinaria por parte de los servicios de dermatología, nefrología, cardiología, oftalmología, psiquiatría infantil, genética médica y neurología pediátrica.

Análisis estadístico: los datos se presentan como estadística descriptiva.

Aspectos éticos. Siguiendo los protocolos establecidos por el hospital, se solicitó a los responsables de los pacientes la autorización para la publicación y estudio de datos clínicos, quienes aceptaron firmando un consentimiento informado.

\section{RESULTADOS}

Se identificaron 12 pacientes con diagnóstico de CET, cinco corresponden al sexo masculino y siete al femenino. Diez de los 12 pacientes (83\%) fueron diagnosticados antes de los dos años, el promedio fue de 16 meses, siendo la variación desde recién nacidos hasta los 72 meses.

Ninguno de los pacientes fueron hijos de padres consanguíneos. Pese al patrón de herencia autosómico dominante, sólo tres (25\%) tenían un familiar de primer grado con la misma entidad.

En la Tabla 2 se describen las características de los pacientes incluidos. De todos, seis presentaron entre dos y tres criterios mayores, cinco presentaron cuatro criterios o más. Mientras que sólo un caso tuvo un criterio mayor.

El signo clínico más frecuente fueron las manchas hipomelanóticas que se observaron en todos los pacientes; 7/12 (58\%) presentaban crisis epilépticas, con una edad media de inicio a 2.3 años, de los cuales $5 / 7$ eran refractarios al manejo antiepiléptico; 10/12 (83\%) de los pacientes tenían algún tipo de alteración cognitivoconductual, entre los que se destacan trastornos del comportamiento, agresividad y trastorno del espectro autista.

Con respecto a otras manifestaciones, hubo dos pacientes con rabdomiomas (16\%), 5/12 (41\%) presentaron alteraciones oftalmológicas y una cuarta parte con fibromas ungueales. En los estudios de imagen se evidenciaron nódulos subependimarios y displasias corticales en 11/12 (91\%) pacientes.
En la actualidad, todos se encuentran en manejo interdisciplinario con las diferentes especialidades pediátricas, terapia física, ocupacional y del lenguaje. Se brindó a los padres ayuda psicológica y asesoramiento genético.

\section{DISCUSIÓN}

El CET se caracteriza por el desarrollo y crecimiento inusual de hamartomas en el cerebro, piel y otros órganos. ${ }^{8}$ Las manifestaciones multisistémicas pueden variar ampliamente, lo que resulta un reto diagnóstico para el clínico, dada que es una condición rara. ${ }^{9}$ A nivel de América Latina no se ha establecido claramente la epidemiología de esta entidad; sin embargo, un estudio desarrollado en Brasil determinó una incidencia de uno por 10,000 a 50,000 nacidos vivos. ${ }^{10}$

En el Hospital Nacional Cayetano Heredia de Perú se describieron 19 casos con una edad promedio al momento del diagnóstico de 5.3 años; de éstos, 88\% debutaron con convulsiones antes de los 11 meses y las lesiones encontradas con mayor frecuencia fueron máculas hipocrómicas (81\%) y angiofibromas faciales (56\%). Dentro de los hallazgos imagenológicos por resonancia magnética del SNC se encontraron nódulos subependimarios en $92 \%$ de los casos y tuberosidades corticales en $31 \% .^{11}$

El CET es un trastorno autosómico dominante con penetrancia casi completa de expresividad variable; de nuestros pacientes un tercio presentan historia familiar. Se han identificado variantes patogénicas causantes de esta entidad en los genes TSC1 y TSC2. Estas proteínas en su estado normal funcionan en un complejo que inhibe la proliferación celular. ${ }^{12} \mathrm{El}$ complejo hamartina-tuberina interviene en múltiples vías de señalización celular, en las que se incluyen las reguladoras del tráfico, crecimiento, proliferación, adhesión y migración celular. ${ }^{13}$

Los casos sin antecedentes familiares pueden representar variantes patogénicas espontáneas o incluso mosaicismos somáticos. ${ }^{14}$ Es importante recalcar que se han identificado casi tres mil variantes patogénicas diferentes que causan CET y una fracción significativa $(\approx 15 \%)$ no tiene una mutación identificada por pruebas genéticas convencionales, por lo que un resultado normal no excluye el diagnóstico de CET. ${ }^{15}$

El diagnóstico de CET se ha basado en manifestaciones clínicas y radiológicas. Se utilizan una serie de criterios clínicos diagnósticos, revisados en el año 2012, donde se propone un diagnóstico definitivo con 
Tabla 2: Descripción de los 12 casos con complejo esclerosis tuberosa.

\begin{tabular}{|c|c|c|c|c|}
\hline Caso & $\begin{array}{l}\text { Edad diagnóstico/ } \\
\qquad \text { sexo }\end{array}$ & Edad actual & Datos clínicos & $\begin{array}{l}\text { Antecedentes de } \\
\text { importancia }\end{array}$ \\
\hline 1 & 6 años/masculino & 7 años & $\begin{array}{l}\text { Cefalea pulsátil con poca respuesta a los analgésicos } \\
\text { Cuadros de agresividad y terrores nocturnos } \\
\text { Cuatro máculas hipomelanóticas de } 0.5 \mathrm{~cm} \text {, con dos lesiones } \\
\text { subependimarias nodulares }\end{array}$ & $\begin{array}{l}\text { Bisabuelos maternos } \\
\text { con fibromas faciales }\end{array}$ \\
\hline 2 & 1 mes/femenino & 5 meses & $\begin{array}{l}\text { Espasmos infantiles } \\
\text { Nueve máculas hipomelanóticas de } 4 \text { a } 5 \mathrm{~mm} \text { (Figura 1) } \\
\text { Displasias focales corticales transmanto y nódulos subependimarios } \\
\text { bilaterales } \\
\text { Rabdomioma cardiaco de } 0.3 \mathrm{~cm} \text { en el ventrículo derecho }\end{array}$ & Ninguno \\
\hline 3 & 1 año/femenino & 16 años & $\begin{array}{l}\text { Epilepsia focal refractaria, asociado a cuadros de auto- y heteroagresión; } \\
\text { discapacidad cognitiva grave. Angiofibromas faciales. Máculas } \\
\text { hipomelanóticas. Hamartoma retiniano en ojo derecho } \\
\text { Nódulos subependimarios y hamartomas corticales supratentoriales }\end{array}$ & $\begin{array}{l}\text { Abuelo paterno con } \\
\text { epilepsia focal, padre } \\
\text { con CET }\end{array}$ \\
\hline 4 & 1 año/masculino & 7 años & $\begin{array}{l}\text { Discapacidad cognitiva } \\
\text { Fibromas ungueales } \\
\text { Epilepsia focal estructural }\end{array}$ & $\begin{array}{l}\text { Hermana con CET, } \\
\text { abuelo paterno con } \\
\text { epilepsia focal }\end{array}$ \\
\hline 5 & 2 años/femenino & 7 años & $\begin{array}{l}\text { Retardo mental } \\
\text { Epilepsia focal. Microcefalia } \\
\text { Parche acrómico de retina } \\
\text { > } 9 \text { máculas hipomelanóticas. Displasia en lóbulos frontal, parietal y } \\
\text { temporal bilateral, además de nódulos subependimarios }\end{array}$ & $\begin{array}{l}\text { Pretérmino, por } \\
\text { hidropesía fetal }\end{array}$ \\
\hline 6 & 3 años/femenino & 8 años & $\begin{array}{l}\text { Trastorno del espectro autista, retraso del lenguaje expresivo } \\
>5 \text { máculas hipomelanóticas }\end{array}$ & Ninguno \\
\hline 7 & 6 meses/femenino & 17 años & $\begin{array}{l}\text { Discapacidad cognitiva moderada } \\
\text { Máculas hipopigmentadas } \\
\text { Angiofibromas faciales } \\
\text { Fibromas ungueales } \\
\text { Tuberosidad cortical en la región frontal y del vértice bilateral, y nódulos } \\
\text { subependimarios calcificados }\end{array}$ & Ninguno \\
\hline 8 & 1 año/masculino & 7 años & $\begin{array}{l}\text { Retardo mental } \\
\text { Epilepsia focal } \\
\text { Hamartomas corticales, nódulos subependimarios en corona radiada y } \\
\text { centros semiovales }\end{array}$ & Ninguno \\
\hline 9 & Nacimiento/femenino & 9 años & $\begin{array}{l}\text { Rabdomiomas cardiacos } \\
\text { Trastorno de conducta } \\
\text { Placa de Shagreen en región frontal. Angiofibromas en cara y dorso de la nariz } \\
\text { Nódulos en uñas de los pies } \\
\text { Túberes cerebrales }\end{array}$ & Ninguno \\
\hline 10 & 4 meses/masculino & 16 años & $\begin{array}{l}\text { Espasmos infantiles, epilepsia focal. Trastorno generalizado del desarrollo } \\
\text { Angiofibromas en cara } \\
\text { Placa de Shagreen en región lumbar } \\
\text { Mancha en hoja de fresno } \\
\text { Múltiples túberes corticales } \\
\text { Parche retiniano } \\
\text { Nódulos de Köenen }\end{array}$ & Ninguno \\
\hline
\end{tabular}


Continúa Tabla 2: Descripción de los 12 casos con complejo esclerosis tuberosa.

\begin{tabular}{|c|c|c|c|c|}
\hline Caso & $\begin{array}{l}\text { Edad diagnóstico/ } \\
\text { sexo }\end{array}$ & Edad actual & Datos clínicos & $\begin{array}{l}\text { Antecedentes de } \\
\text { importancia }\end{array}$ \\
\hline 11 & 1 año/masculino & 18 años & $\begin{array}{l}\text { Retardo mental, trastorno de la conducta } \\
\text { Epilepsia focal } \\
\text { Máculas hipomelanóticas } \\
\text { Múltiples túberes corticales } \\
\text { Angiomiolipoma renal } \\
\text { Hipertensión arterial } \\
\text { Nódulos en encías } \\
\text { Placa de Shagreen }\end{array}$ & Ninguno \\
\hline 12 & 2 meses/femenino & 4 meses & Manchas en hoja de fresno & Ninguno \\
\hline
\end{tabular}

dos criterios mayores, o bien uno mayor más dos menores; mientras que un diagnóstico posible cuando se identifica un criterio mayor o dos o más criterios menores. ${ }^{5,16}$ En la población estudiada, 11 de los 12 pacientes tuvieron un diagnóstico definitivo con más de dos criterios mayores, y sólo uno presentó un posible diagnóstico con un criterio mayor, sin que se pueda descartar que, en el transcurso del tiempo, vaya a presentar otros datos compatibles con CET llevando a un diagnóstico definitivo. Algunos autores proponen estratificar los criterios según el grupo etario, ya que la expresión de síntomas aumenta conforme la edad, lo que explicaría que en la presente serie de casos algunos de los criterios estaban ausentes. ${ }^{17}$

La presentación clínica de la CET varía entre los individuos, incluso entre las familias, siendo importante conocer las principales manifestaciones en diferentes órganos o sistemas. ${ }^{18}$ Por ejemplo, se reporta que las manifestaciones dermatológicas están presentes en aproximadamente $90 \%$ de los pacientes (en la presente serie todos los pacientes las tuvieron); las manchas hipomelanóticas (Figura 1) se caracterizan por adoptar un patrón lineal sobre tronco o miembros, con una configuración oval con un extremo puntiagudo, lo cual se denomina "hojas de Fresno". ${ }^{14}$

Los angiofibromas faciales pueden afectar aproximadamente a $75 \%$ de los pacientes, por lo general entre los cuatro a cinco años, aumentado en número y tamaño a lo largo de la adolescencia y adultez; se definen como hamartomatosis del tejido vascular y conectivo, de localización principal en nariz y región malar, ${ }^{19}$ como se observó en 4/12 de nuestros pacientes. En dos de estos casos se identificaron parches de Shagreen, causados por fibrosis subepidérmica, que aparecen durante la primera década de la vida. Estos consisten en áreas

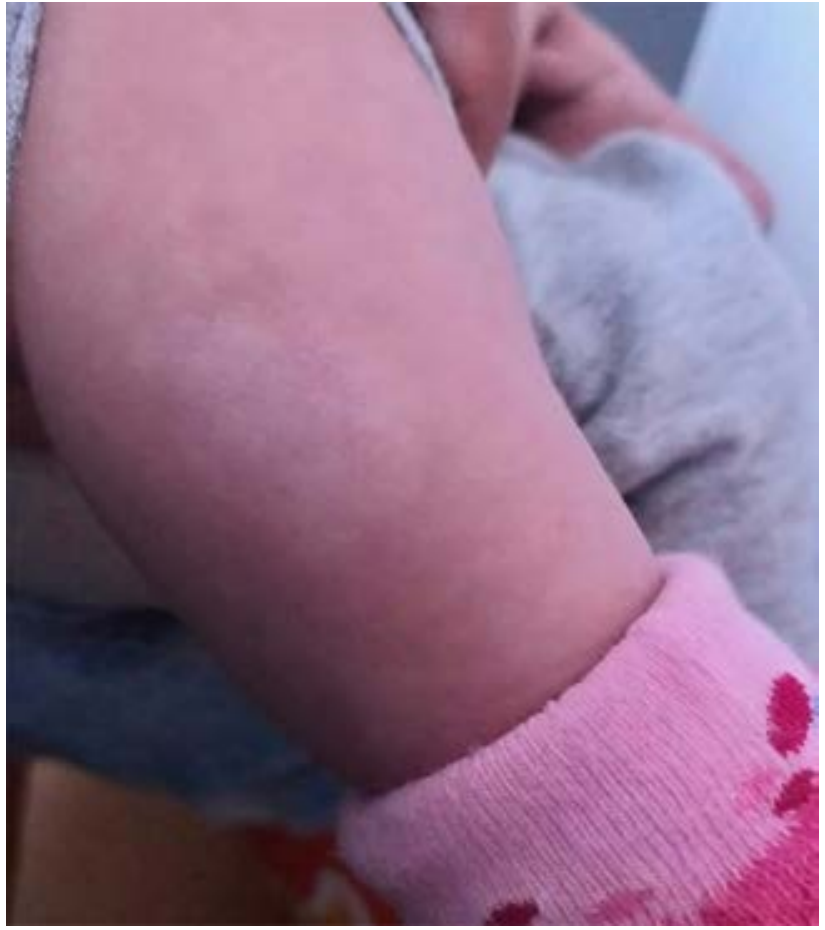

Figura 1: Máculas hipomelanóticas en miembro inferior derecho.

de piel engrosada, con superficie irregular y textura de cáscara de naranja, a menudo ubicados en la cara dorsal del tronco. ${ }^{14,19}$

Durante la segunda década aparecen los fibromas ungueales, llamados tumores de Köenen, que suelen aumentar de tamaño con los años, los cuales se identificaron en una cuarta parte de los pacientes de la presente serie, quienes se encontraban en la etapa escolar o adolescente. ${ }^{19}$ 
Las manifestaciones neurológicas son la principal causa de morbilidad y mortalidad en pacientes con CET, incluyendo las displasias corticales, las cuales se definen como trastornos de la migración neuronal durante el desarrollo embrionario. De los pacientes aquí reportados, la mayoría presentó hamartomas glioneuronales corticales, nódulos subependimarios o líneas de migración radial en la sustancia blanca (Figura 2). ${ }^{20}$

Con respecto al SEGA, los presentan de 5-20\% de los pacientes con CET; son tumores benignos, de crecimiento lento que surgen en el área periventricular. ${ }^{6,21}$ Su detección suele ser después de la segunda década de la vida, causando compromiso neurológico grave, incluyendo hidrocefalia obstructiva, por lo cual se recomienda realizar estudios de imagen de resonancia magnética (IRM) de manera anual o cada tres años; ninguno de los pacientes en esta serie lo presentaron. Por otro lado, con IRM también es posible encontrar lesiones en la sustancia blanca como nódulos, quistes y áreas de gliosis e hipomielinización. ${ }^{22}$

Se ha reportado epilepsia entre $79-90 \%$ de los pacientes, cuyo inicio suele ser entre los tres y ocho meses de edad. Los espasmos infantiles son el tipo de convulsiones más común (hasta en $69 \%$ ); además, se han descrito crisis focales y tónico-clónicas generalizadas. ${ }^{5}$ En nuestra serie, el electroencefalograma (EEG) en la mitad de los pacientes presentó anomalías epileptiformes, incluyendo descargas multifocales, focales y anormalidades generalizadas tipo hipsarritmia. El tipo de epilepsia en la presente correspondió a epilepsia focal y espasmos infantiles, en concordancia con lo publicado en la literatura. ${ }^{5,23}$

El déficit cognitivo es una característica principal del CET; se ha asociado con trastorno del espectro autista, trastorno de déficit de atención e hiperactividad, al igual que otras comorbilidades como depresión, trastorno bipolar, ansiedad, esquizofrenia y psicosis. En nuestros pacientes después del déficit cognitivo predominaron los trastornos conductuales, del espectro autista y cuadros de agresividad. Según la literatura, la prevalencia de estos problemas oscila entre 50-90\% de los pacientes, lo que concuerda con la proporción encontrada en nuestro reporte. ${ }^{24}$

Por otra parte, el rabdomioma cardiaco es una de las características del CET, es considerado el tumor cardiaco primario más común en la infancia temprana, e incluso pueden ser diagnosticados prenatalmente. ${ }^{25}$ Alrededor de $90 \%$ de los pacientes con rabdomioma cardiaco tienen esclerosis tuberosa. ${ }^{26}$ En el grupo de nuestros pacientes sólo dos lo presentaron; en una se detectó en ecografía fetal y se confirmó al nacimiento. Su transformación maligna es mínima e incluso puede haber regresión, por lo que no es necesario algún tratamiento; sin embargo, podrían causar complicaciones como obstrucción intracavitaria, disminución de función miocárdica y arritmias. Se recomienda realizar seguimiento ecocardiográfico cada uno a tres años. ${ }^{27}$

Los problemas renales suelen diagnosticarse en la infancia. Los más frecuentes son angiomiolipomas
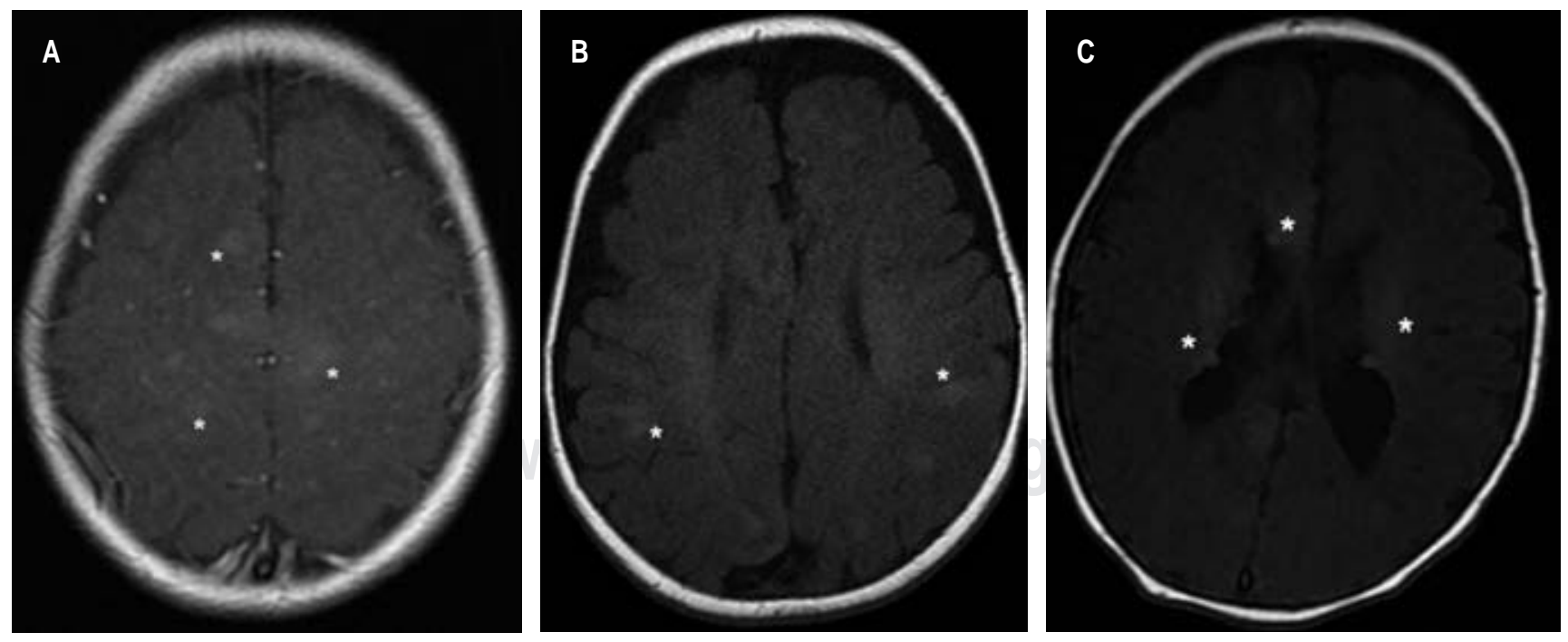

Figura 2: Imágenes de las alteraciones a nivel del sistema nervioso central en el complejo esclerosis tuberosa, señaladas por los asteriscos: A) Túberes corticales multifocales. B) Líneas de migración radial en la sustancia blanca. C) Nódulos subependimarios calcificados. 
(75-85\%), aunque es posible encontrar quistes, linfangiomas y carcinoma de células renales, sobre todo en la edad adulta. Las manifestaciones clínicas incluyen hematuria, dolor lumbar, hipertensión arterial, enfermedad renal crónica y aneurismas. ${ }^{28}$ Sólo uno de los pacientes de esta serie presentó alteraciones renales, pero es importante mantener la vigilancia, ya que este tipo de alteraciones pueden presentarse a mayor edad. $^{29}$

Algunos adultos, predominantemente mujeres entre la tercera y cuarta década de la vida, pueden desarrollar enfermedad pulmonar conocida como linfangioleiomiomatosis (LAM); caracterizada por proliferación e infiltración anormal de las células musculares lisas y una destrucción quística del pulmón. Otro hallazgo pulmonar es la hiperplasia neumocitaria micronodular multifocal, la cual se caracteriza por múltiples nódulos pulmonares compuestos de neumocitos tipo II.

Se considera que hasta la mitad de los pacientes puede presentar manifestaciones oftalmológicas. Los hamartomas retinianos corresponden al hallazgo más común, los cuales pueden ser lesiones planas lisas no calcificadas, lesiones elevadas, multinodulares, calcificada y opacas; ${ }^{30}$ en esta serie $5 / 12$ (41\%) pacientes presentaron alteraciones oftalmológicas, tres con hamartomas retinianos y dos con parches acrómicos de retina.

\section{CONCLUSIONES}

Por último, es importante destacar que, aunque las principales manifestaciones clínicas de los pacientes con CET son problemas dermatológicos y neurológicos, se pueden presentar otras lesiones en otros órganos y sistemas que se presentan a lo largo de la vida, por lo que se requiere manejo y vigilancia de diferentes especialistas. ${ }^{31}$

\section{REFERENCIAS}

1. Lin DDM, Barker PB. Neuroimaging of phakomatoses. Semin Pediatr Neurol. 2006; 13(1): 48-62.

2. Macías DM, Sánchez MN, Cebollero PM, Mandujano AG, Velázquez GG, Soto AV et al. Esclerosis tuberosa. Informe de un caso. Rev Española Patol. 2006; 39(4): 247-249.

3. Wataya-Kaneda M, Tanaka M, Hamasaki T, Katayama I. Trends in the prevalence of tuberous sclerosis complex manifestations: an epidemiological study of 166 japanese patients. PLoS One. 2013; 8(5): 815-835.

4. Wienecke R, Klemm E, Karparti S, Swanson NA, Green AJ, DeClue JE. Reduction of expression of tuberin, the tuberoussclerosis-complex-gene-2 product in tuberous sclerosis complex associated connective tissue nevi and sporadic squamous and basal cell carcinomas. J Cutan Pathol. 2002; 29(5): 287-290.

5. Northrup H, Krueger DA; International Tuberous Sclerosis Complex Consensus Group. Tuberous sclerosis complex diagnostic criteria update: recommendations of the 2012 International Tuberous Sclerosis Complex Consensus Conference. Pediatr Neurol. 2013; 49(4):243-54. doi: 10.1016/j.pediatrneurol.2013.08.001.

6. Curatolo P, Moavero R, de Vries PJ. Neurological and neuropsychiatric aspects of tuberous sclerosis complex. Lancet Neurol. 2015; 14(7): 733-745. Available from: http://dx.doi. org/10.1016/S1474-4422(15)00069-1

7. Barkovich AJ, Guerrini R, Kuzniecky RI, Jackson GD, Dobyns WB. A developmental and genetic classification for malformations of cortical development: Update 2012. Brain. 2012; 135(5): 13481369.

8. Wataya-Kaneda M, Uemura M, Fujita K, Hirata H, Osuga K, Kagitani-Shimono $\mathrm{K}$ et al. Tuberous sclerosis complex: Recent advances in manifestations and therapy. Int J Urol. 2017; 24 (9): 681-691.

9. Ga V, Barkovich J. The phakomatoses. Pediatr Neuroimaging. 2012; 5(2): 563-636.

10. Pascual-Castroviejo I, Pascual-Pascual SI, Velázquez-Fragua R, Viaño J, Carceller F, Hernández-Moneo JL et al. Astrocitoma subependimario de células gigantes en el complejo de esclerosis tuberosa. Presentación de ocho pacientes infantiles. Neurologia. 2010; 25(5): 314-321.

11. Guillén-Pinto D, Gonzales CV, Vidal W, Santivañez C, Vila J, Juárez T et al. Epilepsia en niños atendidos en el Hospital Nacional Cayetano Heredia de Lima, Perú, 2010-2016. Rev Neuropsiquiatr. 2019; 81(4): 217.

12. Jankar tul N, B P, Palange, C V, Purandare. Tuberous sclerosis-A case report. Int J Biomed Res [Internet]. 2015; 6(06): 6. Available from: www.ssjournals.com

13. Henske EP, Scheithauer BW, Short MP, Wollmann R, Nahmias $\mathrm{J}$, Hornigold $\mathrm{N}$ et al. Allelic loss is frequent in tuberous sclerosis kidney lesions but rare in brain lesions. Am J Hum Genet. 1996; 59(2): 400-406.

14. Ebrahimi-Fakhari D, Meyer S, Vogt T, Pföhler C, Müller CSL. Dermatological manifestations of tuberous sclerosis complex (TSC). JDDG - J Ger Soc Dermatology. 2017; 15(7): 695-700.

15. Alva M, Pinto M del pilar G, Daniel R, Olazabal IEQPC, Chocano $M L$, Isabel $M Q$ de $M$. Esclerosis tuberosa: experiencia en pacientes pediátricos en el Hospital Nacional Cayetano Heredia/ Tuberous sclerosis complex: experience in pediatric patients at the Hospital Nacional Cayetano Heredia. Diagnóstico. 2004; 43(5): 199-204.

16. Peron A, Northrup $\mathrm{H}$. Tuberous sclerosis complex. Am J Med Genet Part C Semin Med Genet. 2018; 178(3): 274-247.

17. Randle SC. Tuberous sclerosis complex: a review. Pediatr Ann. 2017; 46 (4): 166-171.

18. Kwiatkowski DJ. Tuberous sclerosis: from tubers to mTOR. Ann Hum Genet. 2003; 67(1): 87-96.

19. Teng JMC, Cowen EW, Wataya-Kaneda M, Gosnell ES, Witman PM, Hebert AA et al. Dermatologic and dental aspects of the 2012 international tuberous sclerosis complex consensus statements. JAMA Dermatology. 2014; 150(10): 1095-1101.

20. Houser OW, McLeod RA. Roentgenographic experience at the Mayo Clinic. In: Gomez MR, ed. Tuberous sclerosis. New York: Raven, 1979: 27-53.

21. Nishio S, Morioka T, Suzuki S, Kira R, Mihara F, Fukui M. Subependymal giant cell astrocytoma: Clinical and neuroimaging features of four cases. J Clin Neurosci. 2001; 8(1): 31-34.

22. Cheng TS. Tuberous sclerosis complex: an update. Hong Kong $J$ Dermatology Venereol. 2012; 20(2): 61-67.

23. Datta AN, Hahn CD, Sahin M. Clinical presentation and diagnosis of tuberous sclerosis complex in infancy. J Child Neurol. 2008; 23(3): 268-273. 
24. Jeste SS, Varcin KJ, Hellemann GS, Gulsrud AC, Bhatt R, Kasari $\mathrm{C}$ et al. Symptom profiles of autism spectrum disorder in tuberous sclerosis complex. Neurology. 2016; 87(8): 766-772.

25. Pajak L, Jin F, Xiao GH, Soonpaa MH, Field LJ, Yeung RS. Sustained cardiomyocyte DNA synthesis in whole embryo cultures lacking the TSC2 gene product. Am J Physiol - Hear Circ Physiol. 1997; 273: 342-343.

26. Salussolia CL, Klonowska K, Kwiatkowski DJ, Sahin M. Genetic etiologies, diagnosis, and treatment of tuberous sclerosis complex. Annu Rev Genomics Hum Genet. 2019; 20(1): 217-240.

27. Karnak I, Alehan D, Ekinci S, Büyükpamukçu N. Cardiac rhabdomyoma as an unusual mediastinal mass in a newborn. Pediatr Surg Int. 2007; 23(8): 811-814.

28. Chen KACLFD Bin. Tuberous sclerosis complex: renal imaging findings. Radiology. 2003; 225(2): 451-456.
29. O'Callaghan FJ, Noakes MJ, Martyn CN, Osborne JP. An epidemiological study of renal pathology in tuberous sclerosis complex. BJU Int. 2004; 94(6): 853-857.

30. Rowley SA, O'Callaghan FJ, Osborne JP. Ophthalmic manifestations of tuberous sclerosis: A population based study. Br J Ophthalmol. 2001; 85(4): 420-423.

31. Amin S, Kingswood JC, Bolton PF, Elmslie F, Gale DP, Harland $C$ et al. The UK guidelines for management and surveillance of tuberous sclerosis complex. Oxford. 2019; 112(3): 171-182.

Conflicto de intereses: los autores declaran que no tienen. 